\title{
Concept of T-Wave Morphology Dispersion
}

\author{
B Acar, G Yi, M Malik \\ Bilkent University, EE. Department, Ankara, Turkey \\ *St. George's Hospital Medical School, London, UK
}

\begin{abstract}
The detection of ventricular repolarisation abnormalities is widely being done using the QT interval measurements. However, there are both technical and theoretical problems with $Q T$ measurements. We propose two robust methods for the quantification of the ventricular repolarisation abnormalities: i) The quantification of the inter-lead morphology differences of the $T$ wave ( $T$ Wave Morphology Dispersion - TMD) ii) The analysis of the $T$ wave wavefront direction with respect to the QRS complex (Total Cosine $R_{-} T o_{-} T$ TCRT).

Sensitivity and specificity of $82 \%(84 \%)$ in supine position and $77 \%(79 \%)$ in standing position were achieved for TMD (TCRT). Both parameters were more reproducible than conventional $Q T$ interval based parameters.
\end{abstract}

\section{Introduction}

The importance of ventricular repolarisation abnormalities in the genesis of ventricular arrhythmias has long been recognised. The analysis of the inter-lead variations of the QT interval, the so called QT Dispersion (QTd), became a popular method for the assessment of ventricular repolarisation. While there are several studies that documented the clinical relevance of QTd, most of them are inconclusive [1]. The poor reproducibility of the QTd, which is mainly due to the problems in the localisation of the $T$ wave end point as well as the presence of $U$ waves and the notched $T$ waves, is the major drawback of QTd. [1-4]. Furthermore, the variation in the QT interval is only a part of the whole picture.

Such concerns led to the attempts to quantify the variation of the ventricular repolarisation patterns. Malfatto et al. and Priori et al. used the principal component analysis (PCA) to quantify the complexity of repolarisation patterns [5-6]. PCA provides a gross measure of the complexity. Kors et al. studied the orientation of the $T$ wave axis [7]. The significance of the relative orientations of the $Q R S$ and the $T$ wave vectors was reviewed by Hurst [8].

We defined two parameters to assess the morphological qualities of ventricular repolarisation patterns and studied their performance in separating normal subjects and the HCM patients. TMD is a measure of the inter-lead $T$ wave morphology variation and TCRT is a measure of the relative wavefront directions of the QRS complex and the $T$ wave.

\section{The method}

All of the measurements are done in a $3 \mathrm{D}$ space, $\mathbf{S}_{\mathbf{3 D}}$, which is constructed by Singular Value Decomposition (SVD) of ECG and is known to represent $98 \%$ of the ECG energy and enhance the ECG signals [9]. Let $\mathbf{M} \in \mathfrak{R}^{8 \times N}$ be the data matrix, whose rows correspond to standard ECG leads I, II, V1, V2, V3, V4, V5, V6. Then,

$$
\begin{aligned}
\mathbf{M} & =\mathbf{U} \Sigma \mathbf{V}^{\mathrm{T}} \\
& =\left[\mathbf{U}_{1} \mathbf{U}_{2}\left\{\begin{array}{cc}
\Sigma_{1} & 0 \\
\mathbf{0} & \Sigma_{2}
\end{array}\right]\left[\begin{array}{c}
\mathbf{V}_{1}^{\mathrm{T}} \\
\mathbf{V}_{2}^{\mathrm{T}}
\end{array}\right]\right. \\
\mathbf{S}_{3 \mathrm{D}} & =\operatorname{span}\left\{\text { columns of } \mathbf{U}_{1} \in \Re^{8 \times 3}\right\}
\end{aligned}
$$

The projection of $\mathbf{M}$ onto $\mathbf{S}_{3 \mathrm{D}}$ is given as $\mathbf{S}=\mathbf{U}_{1}^{\mathrm{T}} \mathbf{M}$. Each column of $\mathbf{S}, \mathbf{S}_{\mathbf{i}}$, represents the ECG vector in $\mathbf{S}_{\mathbf{3 D}}$ and its rows, $\mathbf{d}_{\mathbf{j}}$, are time orthogonal signals.

Approximate QRS complex and $\mathrm{T}$ wave detections are done using the norm of $\mathbf{s}_{\mathbf{i}}, \mathbf{E}_{\mathbf{3 D}}$, and by tracking $\mathbf{S}_{\mathbf{i}}$. Each ECG record is assured to contain only a single beat. The peak of ventricular depolarisation is defined as the time interval where $\mathbf{E}_{3 \mathrm{D}}$ is higher than the $70 \%$ of its 


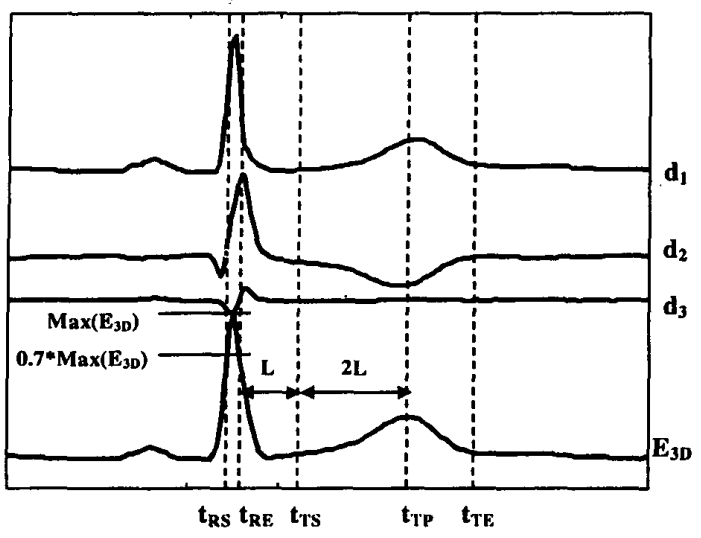

Figure 1 Approximate QRS complex and $T$ wave detection

maximum value. This interval is marked with $t_{R S}$ and $t_{R E}$ in Figure 1. The peak of ventricular repolarisation, $t_{T P}$, is defined as the time instant where $\mathbf{E}_{3 \mathrm{D}}$ is maximum in an interval $(\Gamma)$ starting $50 \mathrm{~ms}$. after $t_{R E}$ and ending at the end of the record. The ventricular repolarisation starting point is defined to be $1 / 3$ of $\left(t_{T P}-t_{R E}\right)$ from $t_{R E}$ (See Figure 1). The rectangular area in $\boldsymbol{S}_{\mathbf{2 D}}$ (the significant subspace of $\mathbf{S}_{3 \mathrm{D}}$ ) which contains $\mathbf{s}_{i, 2 D}$ during the interval $\Gamma$, is divided into 100 equal size cells. Each cell is assigned an index which is equal to the number of consecutive data points in that cell (if a cell is visited twice then it has two distinct indices). Zero indices are discarded. The cell with the lowest index above a threshold is selected. The threshold is

$$
\begin{aligned}
& \text { threshold }=\text { mean }\left(D_{i}\right)+3 \times S T D\left(D_{i}\right) \\
& D_{i}: \mathrm{i}^{\text {th }} \text { nonzero index }
\end{aligned}
$$

The time instant at which $\mathbf{S}_{\mathbf{i}, 2 \mathrm{D}}$ enters this cell is marked as the global repolarization end point, $t_{T E}$. These detections serve our purpose as no exact time domain measurement is necessary.

TCRT is defined as:

$$
\begin{array}{r}
T C R T=\operatorname{mean}\left(\cos \left(\angle\left(\mathbf{s}_{\mathbf{i}}, \mathbf{s}_{T P}\right)\right)\right) \\
t_{R S} \leq \mathbf{i} \leq t_{R E}
\end{array}
$$

It is a measure of the relative directions of the ECG vector during ventricular depolarisation and repolarisation.
The standard ECG channels are reconstructed from $\mathbf{S}$, to obtain morphologically filtered ECG signals: $\hat{\mathbf{M}}=\mathbf{U}_{1} \mathbf{S}$. The $T$ wave section of $\hat{\mathbf{M}}$, which corresponds to the time interval $\left[t_{T S}, t_{T E}\right]$, is redecomposed by SVD:

$$
\begin{aligned}
& \hat{\mathbf{M}}_{\mathbf{T}}=\mathbf{U}_{\mathrm{T}} \boldsymbol{\Sigma}_{\mathbf{T}} \mathbf{V}_{\mathrm{T}}^{\mathbf{T}} \\
& =\left[U_{1, T} U_{2, T}\right]\left[\begin{array}{cc}
\Sigma_{1, T} & 0 \\
0 & \Sigma_{2, T}
\end{array}\right]\left[\begin{array}{l}
V_{1, T}^{T} \\
V_{2, T}^{T}
\end{array}\right] \\
& \mathbf{U}_{1, \mathbf{T}} \in \Re^{8 \times 2}, \Sigma_{1, T} \in \Re^{2 \times 2}, \mathbf{V}_{1, \mathbf{T}} \in \Re^{2 \times K}
\end{aligned}
$$

Thus the influence of the QRS complex on the decomposition is eliminated. Note that $\mathbf{U}_{1, T}$ has two columns, unlike $\mathbf{U}_{1}$ because the most significant $2 \mathrm{D}$ subspace captures most of the energy of $\mathrm{T}$ wave.

Let $\mathbf{W}^{\mathrm{T}}=\mathbf{U}_{1, \mathrm{~T}} \Sigma_{1, \mathrm{~T}}, \mathbf{W} \in \mathfrak{R}^{2 \times 8} . W$ defines the relation between the standard ECG leads and the 2D, normalised sub-space, $V_{1, T}$. Each one of its columns, $w_{j}$ , is the representation of the $\mathrm{j}^{\text {th }}$ ECG lead in this $2 \mathrm{D}$ space. TMD is defined as:

$$
\begin{array}{r}
T M D=\operatorname{mean}\left(\angle\left(\mathbf{w}_{\mathbf{i}}, \mathbf{w}_{\mathbf{j}}\right)\right) \\
\mathbf{i}, \mathbf{j} \in\{I, I I, V 2, V 3, V 4, V 5, V 6\}, \mathbf{i} \neq \mathbf{j}
\end{array}
$$

$\theta_{\mathrm{ij}}=\angle\left(\mathbf{w}_{\mathrm{i}}, \mathbf{w}_{\mathrm{j}}\right)$ represents the $\mathrm{T}$ wave morphology difference between the $i^{\text {th }}$ and $j^{\text {th }}$ ECG leads. If they were identical then $\theta$ would be zero. Thus TMD quantifies the inter-lead $\mathrm{T}$ wave morphology variation. Lead $V 1$ is excluded because its $\mathrm{T}$ wave has usually a different morphology without any physiological background.

A detailed discussion is available in [10].

\section{Data}

10 supine resting and 10 standing position ECGs were recorded in each of 76 normal healthy subjects ( 37 male, aged $38 \pm 10$ years, range $13-59$ years) and 63 patients with hyperthropic cardiomyopathy (HCM) (44 male, aged $39 \pm 14$ years, range 12-71 years). The serial ECGs were recorded consecutively under identical conditions within 3 minutes. Each recording lasted for 10 seconds. We used the median beats calculated from each 10 second recording.

The conventional QT interval based parameters were 
provided by a commercial system (QT Guard, Marquette Medical Systems).

\section{Results}

Table 1 summarises the results. Dichotomy analysis showed that sensitivity and specificity of $82 \%(84 \%)$ in supine position and $77 \%(79 \%)$ in standing position were achievable for TMD (TCRT). The reproducibility of the new parameters is measured using the ratio of individual range (over 10 supine recordings per patient) to the total range (over all subjects and all supine recordings), which is a variability measure. The same analysis is conducted for QTd and QT interval (QTint) also. Table 2 summarises these results.

\section{Discussion}

The new ventricular repolarisation parameters assess different qualities of the $\mathrm{T}$ wave than the conventional time domain based parameters (QTd, etc.) do. This is verified by the low correlation between the parameters. All cross correlation coefficients are below 0.08 .

TMD, which quantifies the $T$ wave morphology deviation between different leads increases in HCM patients. This indicates an increased irregularity of the $\mathrm{T}$ wave morphology in HCM patients. In Figure 2, the T

Table 1 Mean and standard deviation of TMD and TCRT in normal subjects and HCM patients

\begin{tabular}{|c|c|c|c|}
\hline & & Normal & HCM \\
\hline \multirow{2}{*}{$\sum_{G y y}$} & Supine & $10.72^{\circ} \pm 4.78^{\circ}$ & $41.10^{\circ} \pm 26.85^{\circ}$ \\
\cline { 2 - 4 } & Standing & $10.45^{\circ} \pm 6.92^{\circ}$ & $39.39^{\circ} \pm 26.44^{\circ}$ \\
\hline \multirow{2}{*}{ 岂 } & Supine & $0.52 \pm 0.27$ & $-0.35 \pm 0.52$ \\
\cline { 2 - 4 } & Standing & $0.23 \pm 0.41$ & $-0.49 \pm 0.44$ \\
\hline
\end{tabular}

Table 2 Variability of different parameters within 10 consecutive supine recordings (Individual Range / Total Range)

\begin{tabular}{|c|c|c|}
\hline & Normal & HCM \\
\hline TMD & $0.07 \pm 0.06$ & $0.05 \pm 0.05$ \\
\hline TCRT & $0.08 \pm 0.04$ & $0.06 \pm 0.12$ \\
\hline QTd & $0.16 \pm 0.13$ & $0.20 \pm 0.18$ \\
\hline QT int & $0.10 \pm 0.09$ & $0.09 \pm 0.07$ \\
\hline
\end{tabular}
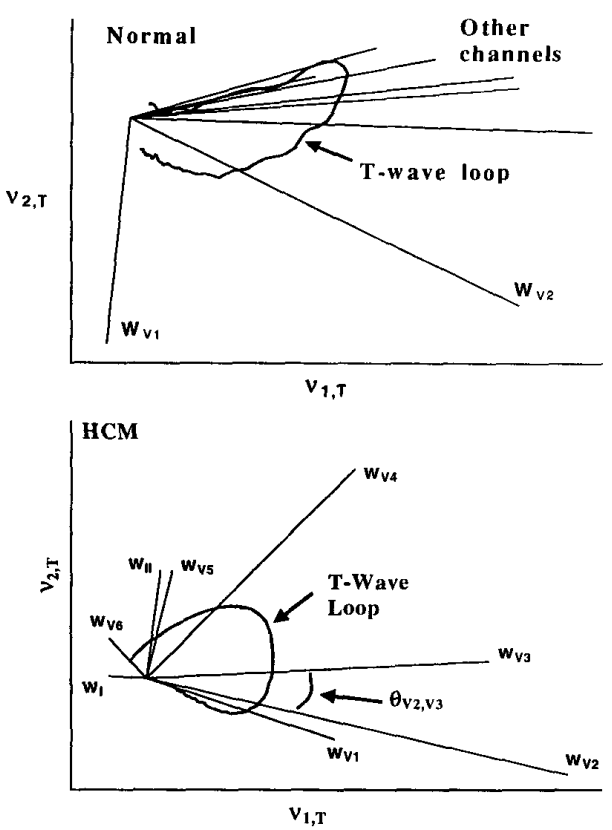

Figure 2 TMD: T-Loop and $\mathbf{w}_{j}$ 's in normal and HCM subjects
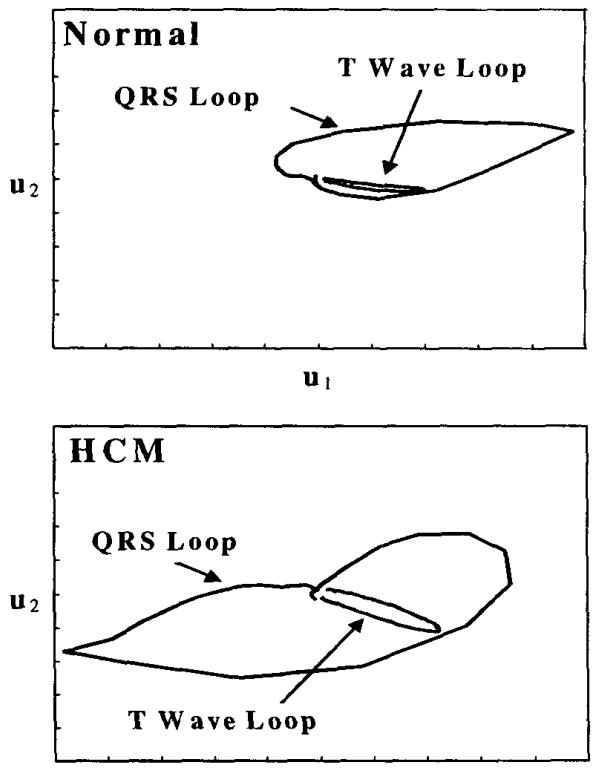

$\mathbf{u}_{\text {। }}$

Figure 3 TCRT: The T-loop and the QRS-loop in normal and HCM subjects 
wave is represented by the loop that the tip of $\mathbf{S}$ traverses during ventricular repolarisation. It is observed that $\mathbf{w}_{j}$ 's are grouped in the normal subject, except $\mathbf{w}_{V 1}$, indicating that the $T$ wave morphologies in different leads are similar, whereas they are far away from each other (dispersed) in the HCM patient.

TCRT, which is a measure of the relative orientation of the ECG vector during ventricular depolarisation and repolarisation, is negative in HCM patients and positive in normal subjects. This indicates that they are aligned in normal subjects and deviate in HCM patients. This can be understood as the alignment and deviation of the $T$ wave loop and the QRS loop, as Figure 3 demonstrates. These loops represent the paths that the ECG vector traverses.

Although the data used in this study was almost noisefree, this method can also be applied to noisy ECG signals. Noise would not affect the new parameters much, as it does conventional time-domain parameters like QTd. This is because both of the new parameters are defined in a minimum dimensional space constructed by SVD which provides a built-in noise immunity and because none of the new parameters require accurate time-domain measurements.

\section{Conclusion}

The new ventricular repolarisation descriptors, TMD and TCRT, exploit the idea of assessing the $T$ wave morphology and the ECG vector orientation. Thus they are grossly different from the conventional time-domain based descriptors, like QTd. The new descriptors

- assess different qualities of the ventricular repolarisation than the conventional descriptors.

- are capable of separating normal subjects and HCM patients.

- do not require accurate time-domain measurements, thus they avoid the inaccuracies associated with time interval measurements.

- have a built-in immunity to noise.

\section{References}

[1] Statters D.J, Malik M, Ward DE and Camm AJ. QT dispersion : Problems of methodology and clinical significance. J. Cardiovasc. Electrophysiol. 1994;5:672-685

[2] Hnatkova K, Malik M, Kautzner J, Gang Y and Camm AJ Adjustment of QT dispersion assessed from 12-lead electrocardiograms for different numbers of analyzed electrocardiographic lead : Comparison of stability of different methods. Br. Heart J. 1994;72:390-396

[3] McLaughlin NB, Campbell RWF and Murray A Comparison of automatic QT measurement techniques in the normal 12-lead electrocardiogram. Br. Heart J. 1995; $74: 84-89$

[4] Murray A, McLaughlin NB, Bourke JP, Doig JC, Furniss SS and Campbell RWF. Errors in manual measurement of QT intervals. Br. Heart J. 1994;71:386-390

[5] Malfatto G, Beria G, Sala S, Bonazzi O and Schwartz PJ Quantitative analysis of $T$ wave abnormalities and their prognostic implications in the idiopathic long QT syndrome. J. Am. Coll. Cardiol. 1994;23:296-301

[6] Priori SG, Mortara DW, Napolitano C, Diehl L, Paganini V, Cantu F, Cantu G and Schwartz PJ. Evaluation of the spatial aspects of $\mathrm{T}$ wave complexity in the long-QT syndrome. Circulation. 1997;96:3006-3012

[7] Kors JA, De Bruyne MC, Hoes AW, Van Herpen G, Hofman A, Van Bemmel JH, Grobbee DE. T axis as an indicator of risk of cardiac events in elderly people. The Lancet. 1998;352:601-605

[8] Hurst JW. Abnormalities of the S-T Segment - 1. Clinical Cardiology 1997;20:511-520

[9] Xue Q and Reddy S. New algorithms for QT dispersion analysis. In: Computers in Cardiology 1996. Indianapolis, USA: IEEE, 1996:293-296

[10] Acar B, Gang Y, Hnatkova K, Malik M. Spatial, Temporal and Wavefront Characteristics of 12-Lead $T$ Wave Morphology. Med. \& Biol. Eng \& Comput. 1999;37:1-11

Address for correspondence:

Burak Acar

Bilkent University

Electrical \& Electronics Eng. Dept.

06533 Ankara, Turkey

email: buraka@ee.bilkent.edu.tr 